\title{
Titrated sedation with propofol or midazolam for flexible bronchoscopy: a randomised trial
}

\author{
G. Clark*,\#, M. Licker", A.B. Younossian" ${ }^{\#}$, P.M. Soccal ${ }^{\#,+}$, J-G. Frey*, \\ T. Rochat", J. Diaper", P-O. Bridevaux" and J-M. Tschopp*
}

ABSTRACT: In this study, we questioned whether propofol provided clinical benefits compared with midazolam in terms of neuropsychometric recovery, safety profile and patient tolerance.

Patients, aged $>18$ yrs, were randomised to receive midazolam or propofol, given by nonanaesthetist physicians to achieve moderate levels of sedation as assessed by the electroencephalographic bispectral index (BIS; between 70 and 85). The primary end-point was the time delay until recovery of the BIS above 90. Other end-points included a neuropsychometric continuous performance test (CPT), serious respiratory adverse events, patient tolerance and physician satisfaction.

Neuropsychometric recovery was improved in the propofol compared to the midazolam group as evidenced by faster normalisation of BIS index (5.4 \pm 4.7 min versus $11.7 \pm 10.2 \mathrm{~min} ; \mathrm{p}=0.001)$ and better results at the CPT. In the midazolam group, $15 \%$ of patients presented profound sedation precluding CPT completion and one patient required mechanical ventilatory support. Patient tolerance was significantly better in the propofol group, whereas the operator's assessment was comparable in both groups.

Compared with midazolam, propofol provided a higher quality of sedation in terms of neuropsychometric recovery and patient tolerance. BIS-guided propofol administration represents a safe sedation technique that can be performed by the non-anaesthesiologist.

KEYWORDS: Bispectral index, bronchoscopy, midazolam, propofol, recovery, sedation

edative techniques using hypnotic and/or analgesic drugs are currently used during flexible bronchoscopy (FB) to facilitate the diagnostic procedure and to improve patient comfort $[1,2]$. Operators often tend to minimise patient discomfort [3-5] and, although FB can be performed without sedation [6-8], a recent survey revealed that $80 \%$ of patients prefer to be sedated during FB [4].

Benzodiazepines are frequently used for sedation given their ease of administration, speed of action and availability of an antidote. Although they undoubtedly enhance operator satisfaction and patient tolerance during FB [1, 2, 4, 9], their major drawbacks are related to numerous drug-drug interactions and variability in metabolic clearance at the level of the CYP3A4 and CYP3A5 (approximately five-fold). Consequently, prolonged sedation, respiratory depression, memory disturbances and other cognitive impairments may occur, particularly in the elderly and patients with liver or renal dysfunction [10].
In contrast to these long-lasting and poorly predictable sedative effects, propofol, a lipid formulation of 2,6 di-isopropylphenol, provides a more rapid onset of sedation after delivery and a faster recovery. Several clinical studies have demonstrated the superiority of propofol compared to midazolam regarding recovery of alertness, memory and motor function [11, 12]. Thus, there is a keen interest in the use of propofol in ambulatory practice. Although propofol is commonly and safely used in gastro-enterological endoscopic procedures, its use by the pneumologist is currently hampered by minimal experience with the drug and lack of collaboration with the anaesthesia team [13-17].

The main purpose of this study was to compare patients' subjective tolerance, recovery of brain function and safety of use after intravenous administration of propofol and midazolam by bolus during FB, using the bispectral index (BIS) as an objective tool for measuring the depth of sedation.
AFFILIATIONS

*Centre Valaisan de Pneumologie, Montana,

${ }^{\text {\#} S e r v i c e ~ d e ~ P n e u m o l o g i e, ~}$

'Service d'Anesthésiologie, and

+Service de Chirurgie Thoracique, Geneva University Hospitals, Geneva, Switzerland.

CORRESPONDENCE

J-M. Tschopp

Centre Valaisan de Pneumologie Centre Hospitalier du Centre du Valais

3963 Crans-Montana

Switzerland

E-mail: jean-marie.tschopp@rsvgnw.ch

Received:

Sept 162008

Accepted after revision:

April 182009

First published online:

May 142009 


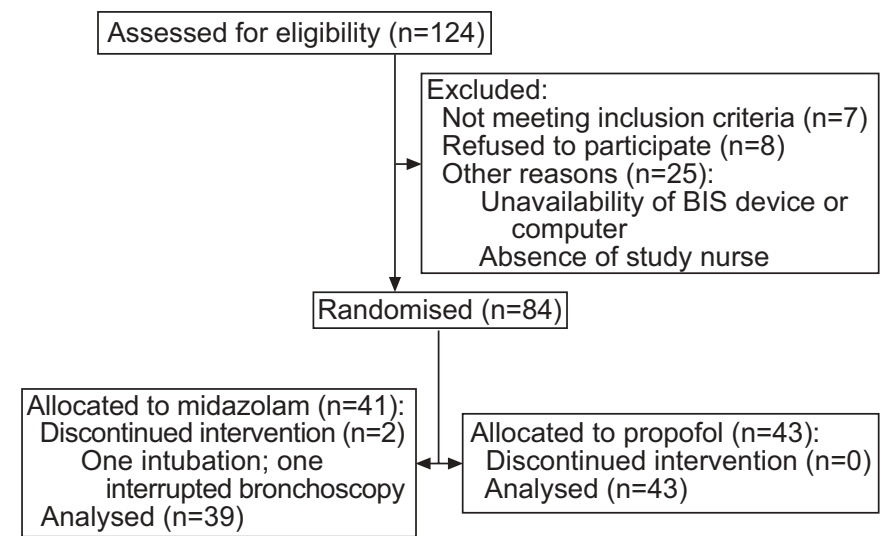

FIGURE 1. Study flow chart. BIS: bispectral index.

\section{METHODS AND MATERIALS \\ Study design}

This was a prospective, randomised and controlled study. The institutional review board at each study site approved the protocol. Written informed consent was obtained from all participants before inclusion in the study.

\section{Subjects}

From May 5, 2006 until June 3, 2007, 124 patients referred for diagnostic FB were recruited at the Centre Valaisan de Pneumologie (Montana, Switzerland) and at the University Hospitals of Geneva (Geneva, Switzerland). Patients undergoing endoscopic procedures such as transbronchial biopsies or advanced techniques (endobronchial ultrasound (EBUS), autofluorescence, etc.) were excluded because of important technical and procedural differences between the two centres and their time-consuming character. Patients aged $\geqslant 18$ and $<80$ years and with an American Society of Anesthesiology (ASA) class of risk I-III were considered eligible for study enrolment. Exclusion criteria included the following items: psychological disorders, female patients of child-bearing age, hypersensitivity or allergy to soya, anaesthetic drugs or benzodiazepine, severe chronic obstructive pulmonary disease (forced expiratory volume in $1 \mathrm{~s}<50 \%$ of predicted value, requirement for oxygen therapy), unstable haemodynamic status (defined as a cardiac frequency $(f C)<60$ or $\geqslant 120 \mathrm{bpm}$ and/or a systolic blood pressure (BP) $<100$ or $>180 \mathrm{mmHg}$ ) and any signs of systemic or pulmonary infection. Other exclusions were patients with predictable difficult upper airways (Mallampati classification score of III or IV).

\section{Study procedures}

Following completion of the pre-procedural assessments, eligible patients were randomly assigned to the midazolam or propofol group using sealed and opaque envelopes in a 1:1 allocation ratio.

All patients were equipped with an intravenous line for fluid infusion and were monitored by continuous electrocardiogram (ECG) for $f C$ and rhythm, pulsed arterial oximetry for arterial oxygen saturation $\left(\mathrm{Sa}_{2} \mathrm{O}_{2}\right)$ and noninvasive blood pressure. Processed electroencephalography (EEG) parameters were acquired with a BIS monitor, using Zip prep surface electrodes, with impedance maintained at less than $5 \mathrm{k} \Omega$ to ensure adequate

\begin{tabular}{|c|c|c|c|}
\hline \multirow[t]{2}{*}{ TABLE 1} & \multicolumn{3}{|c|}{$\begin{array}{l}\text { Baseline clinical characteristics, anthropometric } \\
\text { data and continuous performance test results } \\
\text { before bronchoscopy }\end{array}$} \\
\hline & & Midazolam & Propofol \\
\hline \multicolumn{2}{|l|}{ Subjects $n$} & 39 & 43 \\
\hline \multicolumn{4}{|l|}{ Sex } \\
\hline \multicolumn{2}{|l|}{ Male } & $28(72)$ & $27(63)$ \\
\hline \multicolumn{2}{|l|}{ Female } & $11(28)$ & $16(37)$ \\
\hline \multicolumn{4}{|l|}{ ASA class } \\
\hline \multicolumn{2}{|l|}{1} & $18(46)$ & $14(33)$ \\
\hline \multicolumn{2}{|l|}{ ॥ } & $17(44)$ & $26(60)$ \\
\hline \multicolumn{2}{|l|}{ III } & $4(10)$ & $3(7)$ \\
\hline \multicolumn{4}{|l|}{ Base } \\
\hline \multicolumn{2}{|l|}{ Age yrs } & $55.2 \pm 14.3$ & $57.9 \pm 11.4$ \\
\hline \multicolumn{2}{|l|}{ Weight kg } & $71.6 \pm 12.4$ & $74.9 \pm 15.6$ \\
\hline \multicolumn{2}{|c|}{ Systolic BP mmHg } & $136.5 \pm 18.5$ & $135.8 \pm 16.6$ \\
\hline \multicolumn{2}{|c|}{ Diastolic BP mmHg } & $80.1 \pm 10.1$ & $82.1 \pm 11.7$ \\
\hline \multicolumn{2}{|c|}{$f c$ bpm } & $79.5 \pm 17.8$ & $73.6 \pm 12.3$ \\
\hline \multicolumn{2}{|l|}{$\mathrm{Sa}, \mathrm{O}_{2} \%$} & $96.0 \pm 3.7$ & $95.8 \pm 2.9$ \\
\hline \multicolumn{4}{|l|}{ CPT results } \\
\hline \multicolumn{2}{|l|}{ CE score ${ }^{\#}$} & $8.2 \pm 14.8$ & $8.1 \pm 15.7$ \\
\hline \multicolumn{2}{|c|}{ OE score ${ }^{\#}$} & $13.4 \pm 15.0$ & $10.7 \pm 8.9$ \\
\hline \multicolumn{2}{|c|}{ Reaction time ms } & $463.9 \pm 73$ & $452.9 \pm 128.0$ \\
\hline
\end{tabular}

Data are presented as $n(\%)$ or mean $\pm \mathrm{SD}$, unless otherwise stated. ASA: American Society of Anesthesiology; BP: blood pressure; $f_{C}$ : cardiac frequency; $\mathrm{Sa}_{1} \mathrm{O}_{2}$ : arterial oxygen saturation; $\mathrm{CPT}$ : continuous performance test; $\mathrm{CE}$ : commission error or false hit; $\mathrm{OE}$ : omission error or missed target. \#: $0=$ best score and 100 worst score.

signal quality (AXP-2000 monitor, 3.11 version software; Aspect Medical Systems, Newton, MA, USA). Raw EEG data from two channels $\left(\mathrm{F}_{7}-\mathrm{C}_{\mathrm{Z}}\right.$ and $\left.\mathrm{F}_{8}-\mathrm{C}_{\mathrm{Z}}\right)$ were processed by company proprietary software and the BIS values (calculated for each 4-s epoch) were continuously displayed along with the trend line. A study nurse, blinded to the study drug allocation was trained for proper use of all monitoring devices, including the BIS monitor.

For each procedure, the staff consisted of a chest physician trained in FB (operator), a physician in charge of sedation and two nurses for technical assistance and proper data recording. The operator was unaware of the study drug administration as the syringes and connecting lines were masked. Oxygen was administered only if $\mathrm{Sa}_{1} \mathrm{O}_{2}$ was $<92 \%$.

Before starting sedation, lidocaine was administered topically on the pharynx and upper airways and intravenously (50 mg) to prevent drug-induced pain upon injection. Thereafter, sedation was started by injecting a $4-\mathrm{mL}$ drug bolus $(40 \mathrm{mg}$ of propofol or $2 \mathrm{mg}$ of midazolam). Supplemental doses of drugs (20 $\mathrm{mg}$ of propofol or $2 \mathrm{mg}$ of midazolam) were administered at an interval of $\geqslant 2 \mathrm{~min}$ to achieve and maintain BIS index between 70 and $85[18,19]$. This 2-min time interval between each bolus was based on previous studies [12, 20]. The operator inserted the bronchoscope when the target sedation level was reached. The patient's level of sedation was assessed using the BIS index and the 5-grade Observer Assessment of Alertness/Sedation score (OAAS; $5=$ appropriate verbal response 
TABLE 2 Bronchoscopy and sedation parameters

\begin{tabular}{lccc} 
& Midazolam & Propofol $^{\text {p-value }}$ \\
\hline Subjects $\mathbf{n}$ & 39 & 43 & \\
TIB min & $2.3(1.3)$ & $2.4(1.7)$ & 0.731 \\
FB duration min & $12.2(9.9)$ & $12.4(9.6)$ & 0.368 \\
Recovery time after FB (BIS $>$ 90) & $9.5(15.6)$ & $3.8(7.2)$ & 0.010 \\
Drug dose $\mathbf{m g}$ & $6.2 \pm 2.7$ & $135.1 \pm 71.7$ & \\
\hline
\end{tabular}

Data are presented as median (interquartile range) or mean \pm SD, unless otherwise stated. TIB: time from sedative drug injection to start of bronchoscopy; FB: flexible bronchoscopy; BIS: bispectral index. "\#: Wilcoxon test.

to patient's name, $4=$ lethargic response, $3=$ response only after name is spoken loudly and/or repeatedly, $2=$ response after mild prodding or shaking, $1=$ response after painful stimuli, $0=$ no response at all).

Besides BIS index and OAA/S score, blood pressure, $\mathrm{Sa}_{\mathrm{a}} \mathrm{O}_{2}$, and $f C$, were recorded every 3 min during the procedure and at 5 , $15,30,45$ and 60 min thereafter.

The time necessary to achieve the targeted BIS value after drug injection, the duration of $\mathrm{FB}$, the BIS recovery time (defined as the time to reach a BIS value $>90$ ) and total doses of midazolam and propofol were all noted. The cardiopulmonary safety profile was determined by collecting the following adverse events during FB: hypotension (systolic BP $<100 \mathrm{mmHg}$ or mean arterial blood pressure $(\mathrm{MAP})<60 \mathrm{mmHg}$ ), tachycardia $(f \mathrm{C}>100 / \mathrm{min}$ and $/$ or a variation of $>20 \%$ from baseline value), oxygen desaturation ( $\mathrm{Sa}, \mathrm{O}_{2}$ decrease $<90 \%$ for $>30 \mathrm{~s}$ ), bradycardia ( $\left.\mathrm{HR}<50 / \mathrm{min}\right)$.

At 1 and $24 \mathrm{~h}$ after FB, the operator and patient, both blinded to the allocation group evaluated: 1) the global tolerance to the procedure and 2) the intensity of four key symptoms during FB (pain, nausea, breathlessness and cough) using a visual analogic scale (VAS: $1 \mathrm{~mm}$ : excellent tolerance, $100 \mathrm{~mm}$ very low tolerance).

Recovery of neuropsychometric capacities was also assessed $15 \mathrm{~min}$ and $60 \mathrm{~min}$ after the end of the procedure by a continuous performance test (CPT) [21, 22]. In this standardised computer generated test, the subject was instructed to respond by pressing a computer key, to a specified visual stimulus or target (letters A to Z) appearing randomly on a computer screen. Each letter was shown for $250 \mathrm{~ms}$ and the interval between the two letters was $1 \mathrm{~s}$. Over the course of the test $(7 \mathrm{~min})$, the subject was asked to press a key only when the letter appearing on the screen was the same as the previous one. Each subject was exposed to 335 letters with 170 successful changes. The maximum number of missed targets or omissions errors (OE) possible was 170. The maximum number of false hits or commission errors (CE) possible was $335-170=165$. These values were recorded by the computer and a score ranging from 0 to 100 was calculated by dividing $\mathrm{OE}$ by 170 and $C E$ by 165. A higher score indicated a greater degree of error. These scores define CPT results used in this study. Reaction time (RT) was also recorded. It measured the amount of time between the presentation of the stimulus and the patient's response. A slow reaction time with high $\mathrm{CE}$ and $\mathrm{OE}$ indicates patient inattention.

\section{Study end-points}

The primary end-point was the time delay from the end of the procedure until recovery of BIS $>90$. Previous studies have demonstrated a good correlation between BIS (linear scale from 100 to 0 ) and clinical sedation scores as assessed by the OAAS score during the administration of propofol or midazolam $[18,19,23,24]$.

The secondary end-points were the patient's subjective tolerance, operator evaluation of patient tolerance and cardiopulmonary adverse events rate.

\section{Statistical analysis}

The sample size was calculated for a two-sided significance $\alpha$ level of 0.05 and a power of 0.8 to detect a $35 \%$ difference in recovery time between the two groups. In previous studies comparing midazolam and propofol for outpatient fibroscopy, the mean $\pm \mathrm{SD}$ recovery time was $\sim 10-12 \pm 5 \mathrm{~min}$; thus the minimum number of subjects was $35-40$ per group $[12,20]$. Results are expressed as mean $\pm \mathrm{SD}$ or median (interquartile range). Parametric tests and Wilcoxon test, when appropriate, were used to assess differences between the groups. Analysis of the differences between the midazolam and propofol groups were carried out with robust linear regression models for CPT and tolerance to FB results at 15 and $60 \mathrm{~min}$ and a logistic mixed model for OAAS scores. We applied fixed effects for the sedation group and a random effect for patients. A p-value $<0.05$ was considered to be statistically significant. Recovery time after FB was compared between groups with t-test for unequal variances. It must be emphasised that some patients were unable to complete CPT at $15 \mathrm{~min}$ (six patients) and $60 \mathrm{~min}$ (one patient) after the procedure, and their score of correct, wrong or missed answers is thus not included in the analysis. For these cases, we attributed a reaction time value of $1000 \mathrm{~ms}$ to consider them in the analysis of the reaction time.

We evaluated the overall correlation between OAAS and BIS score with an R-squared value obtained from linear regression models with the study patients as a random effect variable. Statistical analyses were performed with STATA 10 (StataCorp LP; College Station, TX, USA).

\section{RESULTS}

\section{Baseline characteristics}

Out of 124 patients, 84 were randomised (fig. 1). Two patients in the midazolam group were excluded from the final analysis 
because of emergency intubation $(n=1)$ and gag reflex precluding the introduction of the bronchoscope in the trachea $(n=1)$. At baseline there was no difference between both groups (table 1).

The time necessary to achieve the targeted BIS value (70-85) after the injection of the sedative drug before starting the FB (TIB), duration of FB, and mean dose of midazolam and propofol necessary to achieve and maintain the chosen sedation depth according to BIS during FB are shown in table 2.

Overall, the R-squared value between OAAS score and BIS measurements was 0.49 for both groups during the procedure.

\section{Recovery parameters after bronchoscopy}

The EEG recovery time (BIS value $>90$ ) was shorter in the propofol group than in the midazolam group $(5.4 \pm 4.7$ versus $11.7 \pm 10.2$ min; $p=0.001)$. In addition, the rate of patients with a BIS value $>90$ or an OAAS score of 5 (i.e. awake) at any time after the FB was significantly higher after propofol than midazolam sedation (figs 2 and 3 ).

The cognitive recovery evaluated by $\mathrm{CPT}$ at 15 min after FB also showed striking and significant differences for all tested items in favour of the propofol group. At $60 \mathrm{~min}$, no difference was apparent between the groups except the rate of incorrect responses and the reaction time, which remained statistically lower in the propofol group (table 3 ). In the midazolam group only, six (15\%) out of 39 and one (3\%) out of 39 patients at 15 and $60 \mathrm{~min}$ after $\mathrm{FB}$, respectively, were unable to complete the CPT trial because of profound sedation. We performed additional comparisons between midazolam and propofol using nonparametric tests, which yielded a similar interpretation on the differences between groups.

\section{Tolerance}

The immediate tolerance of FB, as assessed by the patient, was better on most items with propofol than midazolam and significantly better on the items "pain", "nausea" and "breathlessness" (table 4). At $24 \mathrm{~h}$ after the procedure, global patient

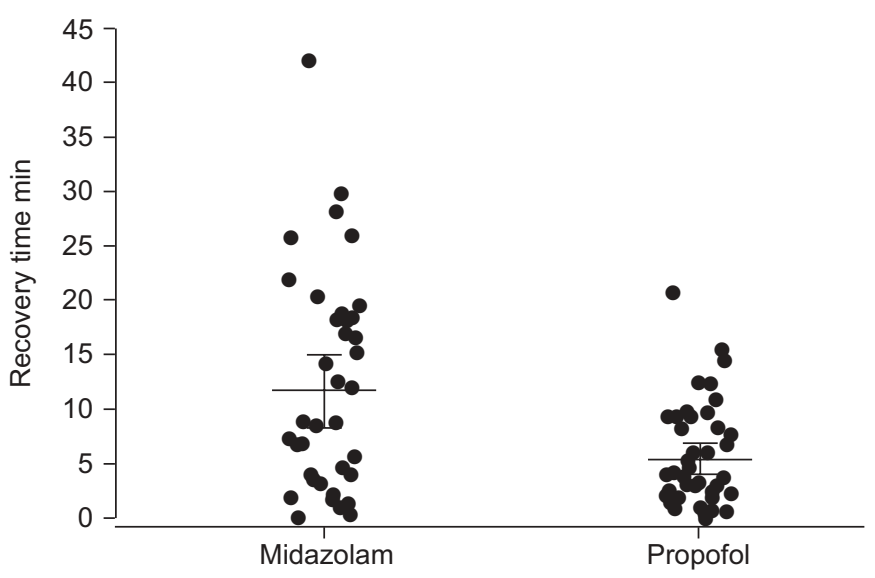

FIGURE 2. Recovery time (min) after flexible bronchoscopy. Horizontal bars are means and whiskers indicate $95 \% \mathrm{Cl}$. Mean (95\% Cl) difference between midazolam and propofol was $380 \mathrm{~s}(170-580 \mathrm{~s})$, in favour of propofol, and $p=0.001$ (t-test with unequal variance). satisfaction was still better in the propofol group, whereas the operator's assessment was similar in the two groups.

\section{Adverse events}

Two patients in the midazolam group required ventilatory support due to oxygen desaturation. A 77-yr-old obese female with moderate chronic obstructive lung disease required intubation. One other patient, with an important gag reflex, needed manual ventilation and the endoscopic procedure was postponed. All other desaturation events were transient and easily corrected with nasal oxygen administration. Apart from the two cases above, there was no difference between the two groups (table 5).

\section{DISCUSSION}

In this trial, we found that propofol is superior to midazolam to enhance patient tolerance, to shorten recovery time and to facilitate return to baseline neurological function after FB. Indeed, recovery time after sedation is impressively faster after propofol compared to midazolam. Both sedation techniques appear safe and enhance the completion rate of the procedure.

Propofol sedation guided by BIS during FB proved to be safe, confirming results from previous studies [12, 14, 15, 17, 20, 25]. However, the good correlation between OAAS score and BIS monitoring during the procedure suggests that the OAAS alone may be sufficient to estimate level of sedation in clinical practice.

The complication rate was similar in both groups, consisting mainly of easily remedied temporary episodes of $\mathrm{O}_{2}$ desaturation and tachycardia. Indeed, $\mathrm{O}_{2}$ had been weaned rapidly at the end of the FB in most cases or within $60 \mathrm{~min}$ for the remaining patients. The causes of $\mathrm{O}_{2}$ desaturation during $\mathrm{FB}$ are manifold (e.g., ventilation-perfusion mismatch due to fluid instillation and excessive secretions) and were not exclusively due to hypoventilation secondary to sedation, as this has also been observed during procedures without sedation [4]. The time to reach the target value of sedation (BIS value 70-85) before starting the FB was not faster for propofol than for midazolam, contrary to what drug pharmacokinetics and some studies might suggest $[10,11,20]$, yet in agreement with other findings [12]. Furthermore, the use of BIS did not induce a change in the average doses of propofol and midazolam $\left(1.9 \mathrm{mg} \cdot \mathrm{kg}^{-1}\right.$ and $0.08 \mathrm{mg} \cdot \mathrm{kg}^{-1}$ respectively) used in our study, when compared with other studies using only clinical scores as assessment of sedation depth [2, 12, 20, 26]. The slower recovery time and the relatively persistent confusional state after midazolam $(15 \%$ of patients were unable to perform the cognitive tests after FB in the midazolam group and none in the propofol group) cannot be explained by an exaggerated use of midazolam, as the dose we used was in the lower range of that recommended in previous guidelines and lower than certain prescription habits in other institutions [2, 27].

We have also shown the intravenous administration of initially lower doses of sedatives by bolus followed by regular increments, to be a simple and safe option, not requiring the use of an infusion pump or target controlled infusion device, as used in other studies [12]. Our study confirms previous findings obtained using clinical sedation scores by incorporating objective measurements of brain activity using the BIS 

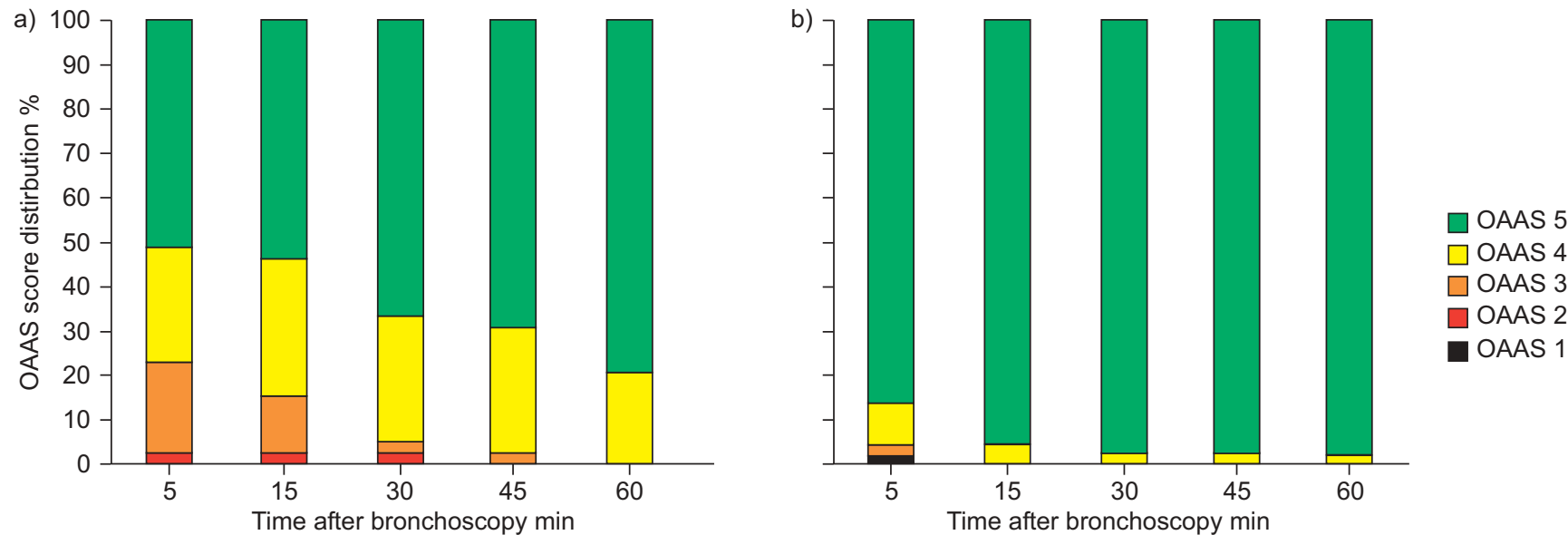

FIGURE 3. Evolution of the Observer Assessment of Alertness/Sedation score (OAAS) score distribution for patients in the a) midazolam and b) propofol groups at different time points after bronchoscopy. Patients with OAAS score $=5$ have full conscious level, patients with OAA/S score $=1$ are asleep and do not react to mechanical stimuli. For the difference between midazolam and propofol, $p=0.004$, derived from mixed logistic model with dichotomised OAAS score $(>4)$, controlling for group, baseline score, time and interaction time group.

technology: the average recovery time was significantly faster for propofol than for midazolam. All patients were alert very quickly after propofol contrasting with the slower time of recovery after midazolam. Within $10 \mathrm{~min}$ of the end of the procedure, nearly $90 \%$ of patients had recovered in the propofol group versus $50 \%$ in the midazolam group. As most endoscopic procedures are performed on an outpatient basis, the use of propofol may increase cost-effectiveness as the duration of monitoring after FB is much shorter when using propofol, counterbalancing its higher cost.

In addition, measuring the objective time of recovery using the $\mathrm{BIS}$ index and performing repeated CPT may enrich further studies, as they allow the objective testing of neurological recovery after sedation [21]. The comparison of both groups showed this clear advantage for propofol on all items tested at $15 \mathrm{~min}$ and for some at $60 \mathrm{~min}$. That study was not designed to evaluate attentional deficits on usual daily tasks such as driving or working after FB. These are most probably only measurable with other more precise and specific neuropsychological tests.

Patient tolerance to the procedure was excellent in both groups, with a slight but significant advantage for propofol. Our study confirms that sedation offers a high degree of satisfaction for the patient without compromising safety.

Interestingly, the assessment related to the tolerance of the procedure differed between patient and operator. In other words, physicians tended in our study to underestimate the tolerance of FB, emphasising the amnesic properties of both drugs.

Some aspects of our study need to be addressed. First, sedation was performed by a second physician trained in the use of propofol and not by a nurse as is usual practice with midazolam. This may result in additional costs. Managing BIS-guided

TABLE 3 Continuous performance test results at 15 and $60 \mathrm{~min}$ after bronchoscopy in both groups

\begin{tabular}{|c|c|c|c|c|}
\hline CPT results & Midazolam $(n=39)$ & Propofol $(n=43)$ & Difference $(95 \% \mathrm{Cl})$ & p-value \\
\hline \multicolumn{5}{|c|}{15 min after bronchoscopy } \\
\hline CE score & $22.5 \pm 13.1$ & $12.2 \pm 10.7$ & $-10.3(-15.7--0.5)$ & $<0.001^{\bullet}$ \\
\hline OE score & $22.7 \pm 16.1$ & $15.2 \pm 13.6$ & $-7.5(-14.2--0.6)$ & $0.032^{\bullet}$ \\
\hline Unable to complete $n$ & 6 & 0 & & \\
\hline Reaction time ms & $486(161)$ & $450(114)$ & & $0.011^{+}$ \\
\hline \multicolumn{5}{|c|}{$60 \mathrm{~min}$ after bronchoscopy } \\
\hline CE score ${ }^{\#}$ & $19.2 \pm 16.7$ & $12.0 \pm 12.9$ & $-7.2(-13.7--0.6)$ & $0.032^{\bullet}$ \\
\hline OE score ${ }^{\#}$ & $16.6 \pm 11.8$ & $13.1 \pm 11.4$ & $-3.4(-0.9-1.7)$ & $0.186^{\bullet}$ \\
\hline Unable to complete $n$ & 1 & 0 & & \\
\hline Reaction time ms & $464(96)$ & 429 (136) & & $0.043^{+}$ \\
\hline
\end{tabular}

Data are presented as mean \pm SD or median (interquartile range), unless otherwise stated. CPT: continuous performance test; CE: commission error or false hit; OE: omission error or missed target. ${ }^{*}: 0=$ best score and 100 worst score; ${ }^{\bullet}$ : differences between groups and p-values derived from robust linear regression using score as dependent variable and group as independent variable; ${ }^{+}$: Wilcoxon test. 
TABLE 4 Tolerance to bronchoscopy as assessed by the patient and the operator with a visual analogic scale (VAS)

\begin{tabular}{|c|c|c|c|c|}
\hline & Midazolam $(n=39)$ & Propofol $(n=43)$ & Difference $(95 \% \mathrm{Cl})$ & p-value ${ }^{\#}$ \\
\hline \multicolumn{5}{|l|}{ Global tolerance } \\
\hline Patient $24 \mathrm{~h}$ & $14.3 \pm 16.7$ & $7.9 \pm 8.7$ & $-6.4(-0.4--12.2)$ & 0.036 \\
\hline Operator & $16.2 \pm 17.4$ & $22.7 \pm 24.9$ & $6.5(-2.9-15.9)$ & 0.171 \\
\hline \multicolumn{5}{|l|}{ Pain } \\
\hline \multicolumn{5}{|l|}{ Nausea } \\
\hline Patient 60 min & $7.7 \pm 13.4$ & $3.2 \pm 4.7$ & $-4.6(-9.0--0.1)$ & 0.047 \\
\hline Patient $24 \mathrm{~h}$ & $8.9 \pm 15.7$ & $4.3 \pm 7.0$ & $-4.6(-10.0-0.8)$ & 0.097 \\
\hline \multicolumn{5}{|l|}{ Breathlessness } \\
\hline Patient 60 min & $13.3 \pm 23.6$ & $4.4 \pm 5.9$ & $-9.0(-16.4--1.2)$ & 0.024 \\
\hline
\end{tabular}

Data are presented as mean $\pm \mathrm{SD}$, unless otherwise stated. VAS: $0 \mathrm{~mm}$ corresponds to excellent tolerance and $100 \mathrm{~mm}$ to very low tolerance. ${ }^{*}: \mathrm{p}$-values derived from robust linear regression using VAS score difference as dependent variable and group as independent variable.

sedation by trained nurses seems to be safe for gastroenterological procedures provided adequate protocols are established in collaboration with the anaesthesia team [28-30]. Another limitation of our study relates to the short time of most procedures. This may preclude conclusions regarding longer procedures such as ultrasound guided trans-bronchial needle aspiration or autofluorescence.

In conclusion, this study shows that, with appropriate training, titrated sedation with propofol using BIS index for FB in an ambulatory setting is safe, can be performed by the nonanaesthetist and allows for greater patient satisfaction. The better neurological recovery with propofol may allow shorter stay in hospital, representing a potential economical benefit. We believe that propofol could be the first choice drug for providing sedation in patients undergoing bronchoscopic procedures.

\begin{tabular}{lccc} 
TABLE 5 & $\begin{array}{l}\text { Adverse events for both groups during } \\
\text { bronchoscopy }\end{array}$ \\
& Midazolam $(\mathbf{n}=\mathbf{3 9})$ & Propofol $(\mathbf{n}=\mathbf{4 3})$ & p value $^{\#}$ \\
\hline Hypotension \% & 0 & $2(4.7)$ & 0.495 \\
Tachycardia \%+ & $11(28.2)$ & $7(16.3)$ & 0.285 \\
Hypoxemia \% & $14(35.9)$ & $15(34.9)$ & 1 \\
Bradycardia \% & 0 & 0 & 1 \\
\hline
\end{tabular}

Data are presented as $\mathrm{n}(\%) .{ }^{*}$ : Fischer's exact test; " : systolic blood pressure $<100 \mathrm{mmHg}$ or mean arterial blood pressure $<60 \mathrm{mmHg}$; ${ }^{+}$: cardiac frequency $>100 \mathrm{bpm}$ and/or a variation of $>20 \%$ from baseline value; ${ }^{\text {s: }}$ arterial oxygen saturation decrease $<90 \%$ for $>30 \mathrm{~s}$, cardiac frequency $<50 \mathrm{bpm}$.

\section{CLINICAL TRIALS}

The study protocol has been registered at clinicaltrials.gov (NCT00839371).

\section{SUPPORT STATEMENT}

The Lancardis Foundation (Martigny, CH 1920, Switzerland), financially supported part of the study.

\section{STATEMENT OF INTEREST}

None declared.

\section{ACKNOWLEDGEMENTS}

We wish to thank J.H. Rubio (Centre Valaisan de Pneumologie, Montana, Switzerland) for helping us to develop the cognitive tests used in this study, N. Praplan (Centre Valaisan de Pneumologie) and M. Metzger (Service de Pneumologie, Geneva University Hospitals, Geneva, Switzerland) for their help in performing the study. Statistical support was provided by D. S. Courvoiser (Clinical Research Center, University of Geneva and Geneva University Hospitals).

\section{REFERENCES}

1 Pickles J, Jeffrey M, Datta A, et al. Is preparation for bronchoscopy optimal? Eur Respir J 2003; 22: 203-206.

2 British Thoracic Society guidelines on diagnostic flexible bronchoscopyThorax 2001; 56: Suppl. 1, i1-i21.

3 Poi PJ, Chuah SY, Srinivas P, et al. Common fears of patients undergoing bronchoscopy. Eur Respir J 1998; 11: 1147-1149.

4 Putinati S, Ballerin L, Corbetta L, et al. Patient satisfaction with conscious sedation for bronchoscopy. Chest 1999; 115: 1437-1440.

5 Rees PJ, Hay JG, Webb JR. Premedication for fibreoptic bronchoscopy. Thorax 1983; 38: 624-627.

6 Colt HG, Morris JF. Fiberoptic bronchoscopy without premedication. A retrospective study. Chest 1990; 98: 1327-1330.

7 Hatton MQ, Allen MB, Vathenen AS, et al. Does sedation help in fibreoptic bronchoscopy? BMJ 1994; 309: 1206-1207. 
8 Pearce SJ. Fibreoptic bronchoscopy: is sedation necessary? Br Med J 1980; 281: 779-780.

9 Maltais F, Laberge F, Laviolette M. A randomized, double-blind, placebo-controlled study of lorazepam as premedication for bronchoscopy. Chest 1996; 109: 1195-1198.

10 Olkkola KT, Ahonen J. Midazolam and other benzodiazepines. Handb Exp Pharmacol, 2008: 335-360.

11 Steinbacher DM. Propofol: a sedative-hypnotic anesthetic agent for use in ambulatory procedures. Anesth Prog 2001; 48: 66-71.

12 Crawford M, Pollock J, Anderson K, et al. Comparison of midazolam with propofol for sedation in outpatient bronchoscopy. Br J Anaesth 1993; 70: 419-422.

13 Heuss LT, Inauen W. The dawning of a new sedative: propofol in gastrointestinal endoscopy. Digestion 2004; 69: 20-26.

14 Carlsson U, Grattidge P. Sedation for upper gastrointestinal endoscopy: a comparative study of propofol and midazolam. Endoscopy 1995; 27: 240-243.

15 Koshy G, Nair S, Norkus EP, et al. Propofol versus midazolam and meperidine for conscious sedation in GI endoscopy. Am J Gastroenterol 2000; 95: 1476-1479.

16 Seifert H, Schmitt TH, Gultekin T, et al. Sedation with propofol plus midazolam versus propofol alone for interventional endoscopic procedures: a prospective, randomized study. Aliment Pharmacol Ther 2000; 14: 1207-1214.

17 Wehrmann T, Kokabpick S, Lembcke B, et al. Efficacy and safety of intravenous propofol sedation during routine ERCP: a prospective, controlled study. Gastrointest Endosc 1999; 49: 677-683.

18 Liu J, Singh H, White PF. Electroencephalographic bispectral index correlates with intraoperative recall and depth of propofolinduced sedation. Anesth Analg 1997; 84: 185-189.

19 Liu J, Singh H, White PF. Electroencephalogram bispectral analysis predicts the depth of midazolam-induced sedation. Anesthesiology 1996; 84: 64-69.
20 Clarkson K, Power CK, O'Connell F, et al. A comparative evaluation of propofol and midazolam as sedative agents in fiberoptic bronchoscopy. Chest 1993; 104: 1029-1031.

21 Riccio CA, Reynolds CR, Lowe $\mathrm{P}$, et al. The continuous performance test: a window on the neural substrates for attention? Arch Clin Neuropsychol 2002; 17: 235-272.

22 Riccio CA, Waldrop JJ, Reynolds CR, et al. Effects of stimulants on the continuous performance test (CPT): implications for CPT use and interpretation. J Neuropsychiatry Clin Neurosci 2001; 13: 326-335.

23 Chernik DA, Gillings D, Laine H, et al. Validity and reliability of the Observer's Assessment of Alertness/Sedation Scale: study with intravenous midazolam. J Clin Psychopharmacol 1990; 10: 244-251.

24 Sandler NA. The use of bispectral analysis to monitor outpatient sedation. Anesth Prog 2000; 47: 72-83.

25 Randell T. Sedation for bronchofiberoscopy: comparison between propofol infusion and intravenous boluses of fentanyl and diazepam. Acta Anaesthesiol Scand 1992; 36: 221-225.

26 Sandler NA, Sparks BS. The use of bispectral analysis in patients undergoing intravenous sedation for third molar extractions. J Oral Maxillofac Surg 2000; 58: 364-368.

27 Williams TJ, Bowie PE. Midazolam sedation to produce complete amnesia for bronchoscopy: 2 years' experience at a district general hospital. Respir Med 1999; 93: 361-365.

28 Heuss LT, Schnieper P, Drewe J, et al. Safety of propofol for conscious sedation during endoscopic procedures in high-risk patients-a prospective, controlled study. Am J Gastroenterol 2003; 98: 1751-1757.

29 Heuss LT, Schnieper P, Drewe J, et al. Conscious sedation with propofol in elderly patients: a prospective evaluation. Aliment Pharmacol Ther 2003; 17: 1493-1501.

30 Heuss LT, Schnieper P, Drewe J, et al. Risk stratification and safe administration of propofol by registered nurses supervised by the gastroenterologist: a prospective observational study of more than 2000 cases. Gastrointest Endosc 2003; 57: 664-671. 